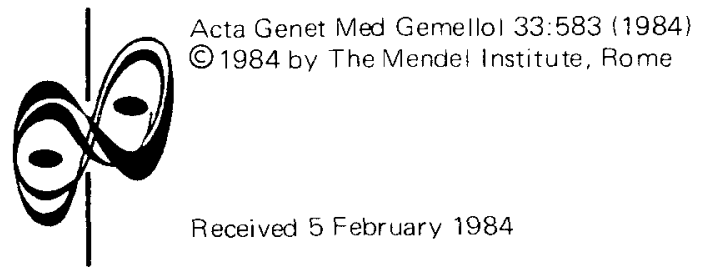

LETTER TO THE EDITOR

\title{
Reply to Dr. Hrubec
}

\author{
M.C. Hannah \\ Epidemiology Unit, Faculty of Medicine, University of Melbourne
}

We are grateful to Dr. Hrubec [5] for emphasising that when the trait of interest is measured imperfectly "the casewise concordance rate" for twins is a biased estimator for the conditional probability of a twin being affected given the cotwin is affected. Dr. Hrubec has previously shown how a secondary ascertainment process with accurate measurement can be used to provide an unbiased estimator; the "proband concordance rate" $[1,3,4]$. As Dr. Hrubec alluded, the proband concordance rate for the trait as measured and the casewise concordance rate are identical.

We would like to make two comments. Firstly, in relation to our study [2], we would like to emphasise that no secondary ascertainment was used (in fact we had "complete ascertainment" for the sample studied); hence, our estimates are valid only for the trait as measured and as Dr. Hrubec rightly points out, they would be subject to bias arising from any imperfect measurement of the trait of interest.

Secondly, we would like to point out that on p. 127 of our article [2] we had meant to imply that the proband (or casewise) concordance rate for the trait measured is independent of the probability of ascertainment; this statement is valid even if the identification of "actually affected" individuals is subject to measurement error.

\section{REFERENCES}

1. Allen G, Hrubec Z (1979): Twin concordance a more general model. Acta Genet Med Gemellol 28:3-13.

2. Hannah MC, Hopper JL, Mathews JD (1983): Twin concordance for a binary trait. I. Statistical models illustrated with data on drinking status. Acta Genet Med Gemellol 32:127-137.

3. Hrubec $Z$ (1973): The effect of diagnostic ascertainment in twins on the assessment of the genetic factor in disease etiology. Am J Hum Genet 25:15-28.

4. Hrubec Z, Allen G (1975): Letter to editor. Methods and interpretation of twin concordance data. Am J Hum Genet 27:808-809.

5. Hrubec Z (1984): Letter to editor. Acta Genet Med Gemellol.

Correspondence: Mr. M.C. Hannah, Fpidemiology Unit, Faculty of Medicine, University of Melbourne, 151 Barry Street, Carlton, Vic 3053, Australia. 